\title{
Effect of Antimicrobial Photodynamic Therapy as an Adjunct to Nonsurgical Treatment of Deep Periodontal Pockets: A Clinical Study
}

\author{
Abdul Ahad ${ }^{*}$, Arundeep Kaur Lamba², Farrukh Faraz², Shruti Tandon², Kirti Chawla², Neha Yadav² \\ ${ }^{1}$ Department of Periodontics, Dr. Ziauddin Ahmad Dental College, Aligarh Muslim University, Aligarh, India \\ ${ }^{2}$ Department of Periodontics, Maulana Azad Institute of Dental Sciences, Bahadur Shah Zafar Marg, New Delhi, India
}

\author{
*Correspondence to \\ Abdul Ahad, MDS; Department of \\ Periodontics, Dr. Ziauddin Ahmad \\ Dental College, Aligarh Muslim \\ University, Aligarh - 202002, India. \\ Tel: +91-9582439639; \\ Email: alig.maids@gmail.com
}

Published online 27 October 2016

\begin{abstract}
Introduction: Deep periodontal pockets pose a great challenge for nonsurgical periodontal treatment. Scaling and root planing (SRP) alone may not suffice in cases where surgical therapy cannot be undertaken. Various recent studies have suggested the use of antimicrobial Photodynamic Therapy (aPDT) for the management of periodontal infections. The aim of this study was to evaluate the effects of using aPDT along with SRP, compared to SRP alone for the management of deep periodontal pockets.

Methods: Thirty patients with chronic periodontitis, who met the criteria of having periodontal pockets with depth $\geq 6 \mathrm{~mm}$ and bleeding on probing (BOP) in at least 2 different quadrants were included. After SRP, one quadrant was randomly selected for aPDT (test), while another served as control. Clinical parameters i.e. plaque index (PI), modified sulcular bleeding index $(\mathrm{mSBI})$, probing depth (PD) and clinical attachment level (CAL) were measured at baseline, 1 month and 3 months post-treatment intervals.

Results: All clinical parameters significantly improved in both groups after 1 and 3 months. At 1-month interval, inter-group difference in mean change was statistically significant $(P<0.05)$ in terms of $\mathrm{mSBI}(0.85 \pm 0.41$ in test vs $0.54 \pm 0.47$ in control group $)$ and $\mathrm{PD}$ $(1.77 \pm 0.86$ in test vs $1.3 \pm 0.95$ in control group). At 3 months interval, no statistically significant difference was observed between test and control groups except in terms of $\mathrm{mSBI}(0.97 \pm 0.45$ in test vs $0.73 \pm 0.42$ in control group).

Conclusion: aPDT appears to play an additional role in reduction of gingival inflammation when used along with nonsurgical mechanical debridement of deep periodontal pockets. Keywords: Antimicrobial therapy; Photodynamic therapy; Periodontal pocket; Periodontitis; Diode laser.
\end{abstract}

\section{Introduction}

Photodynamic therapy (PDT), also known as phototherapy, photo radiation therapy or photo chemotherapy has been developing rapidly within various medical specialties since last few decades. PDT has been used in the treatment of premalignant lesions and recurrent tumours after previous surgery or chemotherapy. ${ }^{1}$ The therapeutic use of photodynamics continues to expand for the management of various non-oncological diseases and superficial infections like candidiasis and herpes virus infections. Accordingly, the terminology used for treatment changes from PDT associated with treating oncological diseases to photodynamic antimicrobial chemotherapy or antimicrobial photodynamic therapy (aPDT) in treating localized bacterial, fungal, viral and yeast infections. ${ }^{2}$ aPDT has found its role in periodontal therapy as well. ${ }^{3}$ The main objective of the periodontal therapy is to stop the progress of disease by reduction of plaque biofilm, as well as eliminating the factors that favour its deposition. Although, mechanical removal of plaque biofilm by scaling and root planing (SRP) is an essential part of periodontal therapy, complete removal of plaque and plaque retentive factors is not always possible especially in less accessible sites such as deeper pockets and furcation areas. ${ }^{3,4}$ Additionally, bacteria that penetrate the gingival tissue cannot be eliminated by mechanical instrumentation.

To supplement the armament of mechanical debridement, different adjunctive modalities have been evaluated, including the local and systemic use of antibiotics. However, biofilm structure of the dental plaque confers significant resistance to bacterial species against antibiotics. On systemic administration, availability of insufficient concentration of the drug in gingival crevicular fluid (GCF), disturbance of intestinal micro flora and development of antibiotic resistant strains are the major disadvantages. ${ }^{5,6}$

Please cite this article as follows: Ahad A, Lamba AK, Faraz F, Tandon S, Chawla K, Yadav N. Effect of antimicrobial photodynamic therapy as an adjunct to nonsurgical treatment of deep periodontal pockets: a clinical study. J Lasers Med Sci. 2016;7(4):220-226. doi:10.15171/ jlms.2016.39. 
Therefore, the development of alternative antibacterial therapeutic modalities such as aPDT becomes important for the effectiveness of periodontal treatment.

The use of aPDT for the management of inflammatory periodontal disease is based on the concept that a photosensitizer, usually a phenothiazine compound, that absorbs light, can be preferentially taken up by bacteria, and subsequently activated by a light of appropriate wavelength, in the presence of oxygen, to generate singlet oxygen and free radicals that are cytotoxic to microorganisms. These cytotoxic species can damage plasma membranes and DNA, resulting in cell death. Cell membranes are destroyed by multiple mechanisms, like lipid peroxidation, inactivation of membrane transport system and inhibition of plasma membrane enzyme activities. ${ }^{7}$ Phenothiazine compounds (e.g. toluidine blue $\mathrm{O}$ and methylene blue), which bear a positive charge, can directly target both gram-negative and gram-positive bacteria. The positive charge promote the binding of photosensitizer to the outer bacterial membrane, inducing localized damage, which favours its penetration. It is specifically the pathogenic bacteria that are destroyed by aPDT, sparing the host cells. Unsaturated fatty acids in the bacterial membranes are particularly susceptible to damage by oxygen radicals, as these do not have the body's own enzyme defences as is the case with healthy cells. Antimicrobial PDT appears to significantly reduce inflammation without antibiotics and without surgical intervention while providing maximum therapeutic safety. As aPDT utilizes cold laser light for activation of photosensitizer, it is safe for human tissue as well as dental materials. On the other aspect, subgingival curettage by thermal lasers can damage root surfaces and/or carbonize soft tissues, which may inhibit reattachment of junctional epithelium. Preferential uptake of photosensitizers by bacteria, precise direction of laser light using optical fibres, broad spectrum effect and no development of resistance on repeated application are the major advantages of aPDT in periodontal treatment. Various clinical trials have evaluated the effect of aPDT in treatment of periodontal infections, but results have largely been controversial., 8

There are various limitations of nonsurgical therapy in deep periodontal pockets. In addition to being less accessible for mechanical debridement, deeper sites are difficult to maintain and harbour more anaerobic microorganisms than shallower pockets. ${ }^{10}$ Therefore treatment outcome differs between shallow and deep periodontal pockets even with the same treatment modality. ${ }^{11}$ Potential scope of aPDT in deeper pockets and less accessible sites led to the conception of the present study, with the aim to evaluate the effect of adjunctive use of aPDT in treatment of deep periodontal pockets compared to conventional SRP alone.

\section{Methods}

Study Design

This split mouth, single blinded, randomized controlled clinical trial was approved by the Ethical Committee of
Maulana Azad Institute of Dental Sciences, New Delhi, on 27th September 2012 and conducted according to the principles outlined in the Declaration of Helsinki (1975), on experimentation involving humans as revised in 2000. This clinical trial has been registered in the Iranian Registry of Clinical Trials (IRCT) (registration ID: IRCT2016090329668N1). This trial involved 30 adult patients (age $>18$ years) of either sex who met the inclusion criteria and agreed to sign the informed consent form. The patients were selected randomly from the Outpatient Department of Periodontics at Maulana Azad Institute of Dental Sciences, New Delhi.

Inclusion criteria were the presence of at least 2 teeth in different quadrants with probing depth (PD) $\geq 6 \mathrm{~mm}$, and bleeding on probing (BOP). Exclusion criteria were (1) Systemic conditions which could influence the outcome of study, like uncontrolled diabetes mellitus; (2) Pregnant or lactating women; (3) Any history of smoking or smokeless tobacco use; (4) History of antibiotic use in the last 3 months; (5) Undergoing regular treatment with immunosuppressants, non-steroidal anti-inflammatory drugs, steroids, anticonvulsants, anticoagulants or calcium channel blockers; and (6) Presence of carious lesion or restoration on the root surface.

Patients who fulfilled the inclusion criteria, were given the information sheet and explained the treatment methods and potential risks and benefits. Thirty patients (21 males and 9 females) signed the informed consent form following which detailed history and examination was carried out and recorded.

\section{Recording of Clinical Parameters}

The following clinical parameters were recorded at 4 sites in all teeth using mouth mirror, explorer and calibrated periodontal probe (UNC-15, Hu-Friedy, Chicago, IL). (1) Plaque index (PI) - Sillness \& Loe. (1964), (2) modified sulcular bleeding index (mSBI) - Mombelli et al (1987), (3) probing depth (PD) and (4) clinical attachment level (CAL).

Each patient received thorough oral hygiene instructions. They were also trained in modified Bass technique. Out of the sites with $\mathrm{PD} \geq 6 \mathrm{~mm}$ in different quadrants, 2 sites with the deepest PD were selected for evaluation, and acrylic stents were fabricated for the measurement of $\mathrm{PD}$ and CAL. The measurements of PD and CAL were repeated for the selected sites after placing the acrylic stents. A vertical groove was made in the stent at the site corresponding to the deepest PD to reproduce the position and angulation of probe at subsequent visits (Figure 1A).

Intra-examiner Reliability

Four patients, not related to study, each with two contralateral teeth with $\mathrm{PD}>6 \mathrm{~mm}$, were used to calibrate the examiner. The examiner evaluated the patients at two visits that were separated by 3 days. Calibration was accepted if the data at baseline and 3 days later were the same in $>90 \%$ cases. This procedure was repeated periodically during the study period. 


\section{Scaling and Root Planing}

Thorough full mouth supragingival and sub-gingival scaling was performed using ultrasonic scaler (BonART-P6, BonART Co. Ltd., Taiwan) and hand instruments. Root planing was done with Gracey curettes, until the operator felt that root surfaces were hard and smooth. Normal saline $(0.9 \% \mathrm{NaCl} \mathrm{w} / \mathrm{v})$ was used to irrigate the operative field.

\section{Antimicrobial Photodynamic Therapy}

By flip of a coin, one quadrant was selected for aPDT (test), while another quadrant served as the control. After SRP, haemostasis was achieved and PDT was performed in the test quadrant. Phenothiazine chloride $\left(\mathrm{HELBO}^{\mathbb{R}}\right.$ Blue Photosensitizer, Bredent Medical, Germany) was injected in the bottom of the pocket using a blunt cannula until it appeared flowing out over the gingival margin (Figure 1B). After leaving the dye in the pocket for a duration of 3 minutes, the pocket was irrigated with normal saline to remove the excess dye, as it could act as an optical shield during laser irradiation (Figure 1C). ${ }^{12,13}$ Laser light application was performed circumferentially at six sites per tooth for 1 minute (10 seconds each for mesio-buccal, buccal, disto-buccal, mesio-oral, oral and disto-oral surfaces of tooth), at wavelength of $660 \mathrm{~nm}$, and power density of $100 \mathrm{~mW} / \mathrm{cm}^{2}$. Fiber-optic tip $\left(\mathrm{HELBO}^{\circledR}\right.$ 3D pocket probe, Bredent Medical, Germany) of $0.6 \mathrm{~mm}$ diameter was inserted to the depth of pocket, followed by illumination for 10 seconds. It was then moved to the next site, till all 6 sites around a tooth were exposed to the laser light (Figure 1D). Application times of both the photosensitizer and laser light were monitored by a time controller (HELBO ${ }^{\circledR}$ T-Controller, Bredent Medical, Germany). All patients were recalled after 1 week to check their adherence to oral hygiene instructions. Clinical parameters were recorded again, 1 month and 3 months after the first application of aPDT (Figure 1E).

\section{Results}

A total of 60 sites in 30 patients (21 males and 9 females) were selected and randomly assigned to either SRP alone (control) or SRP along with aPDT (test). For statistical analysis, the SPSS version 10.0 (SPSS Inc., Chicago, IL, USA) was used. Values were reported in terms of number $(\mathrm{n})$, mean $(\mu)$ and standard deviation $(\sigma)$. For the comparison of mean values between treatment groups, Student's $t$ test was used. The analysis of variance (ANOVA) was used for comparison of mean values within each treatment group at different time intervals. The level of significance was taken at $5 \%(P<0.05)$.

The average age of patients was $38.67 \pm 10.52$ years. None of the clinical parameters showed significant inter group difference at baseline (Table 1).

Healing was uneventful in all cases and no adverse effects were reported by any of the subjects after SRP or PDT. There were no drop-outs in the patient sample during the course of the study. Mean values of clinical parameters and difference at 1 month and 3 months interval are
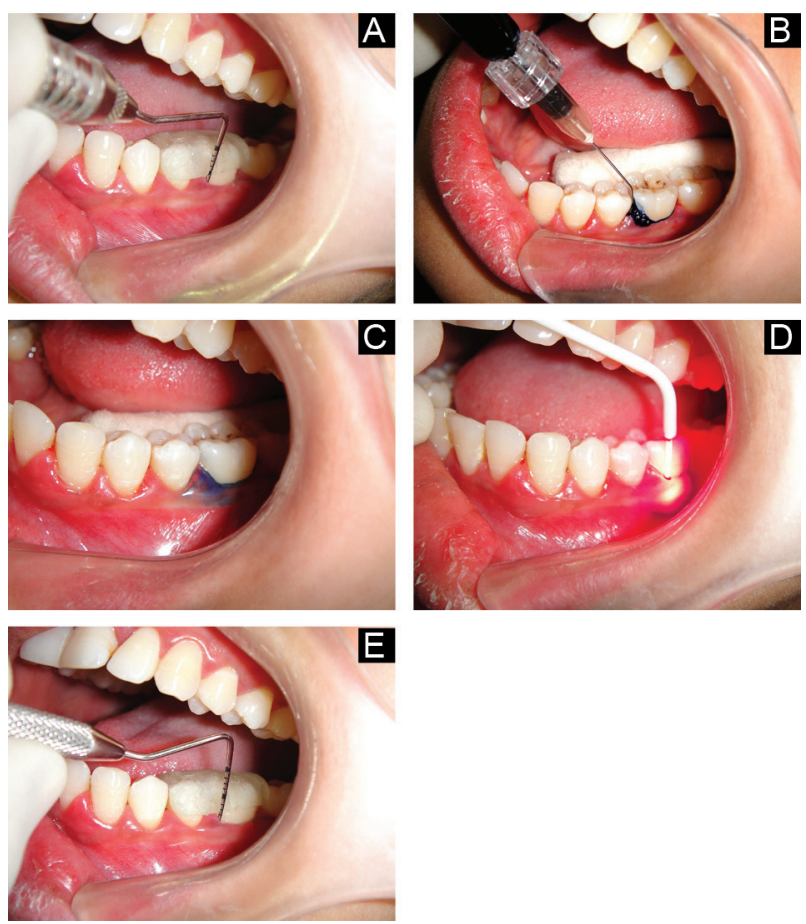

Figure 1. (A) Probing of Test Site at Baseline. (B) Injection of Photosensitizer Dye Into Periodontal Pocket. (C) Test Site After Irrigation With Normal Saline. (D) Illumination of Photosensitizer by Fibre Optic Tip. (E) Probing of Test Site After 3 Months.

shown in Table 2.

Plaque Index

Mean PI reduction at 1 month was found to be $0.43 \pm 0.5$ in the control group and $0.48 \pm 0.60$ in test group, and after 3 months it was found to be $0.47 \pm 0.53$ and $0.62 \pm 0.47$ respectively. The comparison of the mean PI reduction between two groups at 1 month and 3 months was done using Student's $t$ test. No significant inter-group difference was observed after 1 and 3 months.

Modified Sulcular Bleeding Index

The mean mSBI reduction at 1 month was found to be $0.54 \pm 0.47$ in the control and $0.85 \pm 0.41$ in the test group, and after 3 months, it was found to be $0.73 \pm 0.42$ and $0.97 \pm 0.45$ respectively. The comparison of mean $\mathrm{mSBI}$ reduction between the two groups at 1 and 3 months was done using Student's $t$ test. At both 1 and 3 months follow up, combination of SRP+aPDT was significantly more

Table 1. Comparison of Mean Values of Parameters at Baseline

\begin{tabular}{lccc}
\hline Parameters & $\begin{array}{c}\text { SRP } \\
(\text { Mean } \pm \text { SD) }\end{array}$ & $\begin{array}{c}\text { SRP }+ \text { aPDT } \\
(\text { Mean } \pm \text { SD })\end{array}$ & P Value \\
\hline PI & $1 \pm 0.45$ & $1.09 \pm 0.47$ & 0.07 \\
$\mathrm{mSBI}$ & $1.17 \pm 0.38$ & $1.28 \pm 0.41$ & 0.06 \\
$\mathrm{PD}(\mathrm{mm})$ & $7.53 \pm 1.31$ & $7.90 \pm 1.71$ & 0.26 \\
$\mathrm{CAL}(\mathrm{mm})$ & $8.46 \pm 1.81$ & $8.7 \pm 1.91$ & 0.27 \\
\hline
\end{tabular}

Abbreviations: PI: plaque index, mSBI: modified sulcular bleeding index, PD: probing depth, CAL: clinical attachment level.

Values are presented as mean \pm standard deviation. 
Table 2. Mean Values of Clinical Parameters and Differences at 1 Month and 3 Months

\begin{tabular}{|c|c|c|c|c|c|}
\hline Parameter & Baseline & 1 Month & Difference (0-1 Month) & 3 Months & Difference (0-3 Months) \\
\hline \multicolumn{6}{|c|}{$\mathbf{P I}$} \\
\hline Control & $1.00 \pm 0.45$ & $0.57 \pm 0.40$ & $0.43 \pm 0.50$ & $0.46 \pm 0.17$ & $0.47 \pm 0.53$ \\
\hline Test & $1.09 \pm 0.47$ & $0.54 \pm 0.41$ & $0.48 \pm 0.60$ & $0.47 \pm 0.18$ & $0.62 \pm 0.47$ \\
\hline$P$ value & 0.02 & 0.25 & 0.59 & 0.32 & 0.12 \\
\hline \multicolumn{6}{|c|}{ mSBI } \\
\hline Control & $1.17 \pm 0.38$ & $0.63 \pm 0.40$ & $0.54 \pm 0.47$ & $0.46 \pm 0.27$ & $0.73 \pm 0.42$ \\
\hline Test & $1.28 \pm 0.41$ & $0.43 \pm 0.42$ & $0.85 \pm 0.41$ & $0.31 \pm 0.30$ & $0.97 \pm 0.45$ \\
\hline$P$ value & 0.06 & 0.02 & $0.002^{a}$ & 0.03 & $0.015^{\mathrm{a}}$ \\
\hline \multicolumn{6}{|c|}{ PD (mm) } \\
\hline Control & $7.53 \pm 1.31$ & $6.17 \pm 1.20$ & $1.30 \pm 0.95$ & $5.37 \pm 0.85$ & $2.13 \pm 1.22$ \\
\hline Test & $7.90 \pm 1.71$ & $6.27 \pm 1.44$ & $1.77 \pm 0.86$ & $5.43 \pm 1.36$ & $2.37 \pm 1.10$ \\
\hline$P$ value & 0.26 & 0.64 & $0.01^{\mathrm{a}}$ & 0.77 & 0.47 \\
\hline \multicolumn{6}{|c|}{$\mathrm{CAL}(\mathrm{mm})$} \\
\hline Control & $8.47 \pm 1.81$ & $7.53 \pm 1.52$ & $0.97 \pm 0.96$ & $6.73 \pm 1.31$ & $1.73 \pm 1.26$ \\
\hline Test & $8.70 \pm 1.91$ & $7.43 \pm 1.43$ & $1.27 \pm 1.11$ & $6.67 \pm 1.45$ & $2.10 \pm 1.35$ \\
\hline$P$ value & 0.54 & 0.74 & 0.12 & 0.83 & 0.18 \\
\hline
\end{tabular}

Abbreviations: PI: plaque index, mSBI: modified sulcular bleeding index, PD: probing depth, CAL: clinical attachment level.

Values are presented as mean \pm standard deviation.

a Statistically significant difference between test and control groups.

effective $(P<0.05)$ in reduction of $\mathrm{mSBI}$ as compared to SRP alone.

\section{Probing Depth}

The mean PD reduction at 1 month was found to be $1.30 \pm 0.95$ in the control group and $1.77 \pm 0.86$ in the test group and at 3 months it was found to be $2.13 \pm 1.22$ and $2.37 \pm 1.10$ respectively. Inter-group difference was statistically significant at 1 month, but not after 3 months. This result implies that although the PD decreased significantly in both treatment groups compared to baseline, a combination of SRP and aPDT was more effective in the reduction of $\mathrm{PD}$ at 1 month after treatment. However, at 3 months post treatment intervals both group appeared to have similar reduction in PD.

\section{Clinical Attachment Level}

The mean CAL gain at 1 month was found to be $0.97 \pm 0.96$ $\mathrm{mm}$ in the control group and $1.27 \pm 1.11 \mathrm{~mm}$ in the test group and at 3 months, it was found to be $1.73 \pm 1.26 \mathrm{~mm}$ and $2.10 \pm 1.35 \mathrm{~mm}$ respectively. The results indicate that the CAL improved significantly from baseline to 1 and 3 months post treatment interval in both groups. However, SRP and SRP \pm aPDT seems to be equally effective in this regard as the inter-group difference was statistically not significant $(P>0.05)$.

\section{Discussion}

The traditional concept of treatment for chronic periodontitis aims to stop the inflammatory process through sub-gingival biofilm removal. It includes plaque control measures and nonsurgical SRP followed by surgical therapy wherever indicated. Since deep periodontal pockets have more anaerobic microbiota and respond less favour- ably to SRP than shallower ones, it is relevant to evaluate the effect of adjunctive modalities in nonsurgical treatment of such cases.

Deep periodontal pockets were selected as these are difficult to be completely debrided by SRP alone due to limited access. Although post-SRP good oral hygiene can reduce the numbers of pathogenic bacteria subgingivally, but only in pockets less than $5 \mathrm{~mm}$ deep. ${ }^{14,15}$ Generally, these sites are subjected to open flap debridement for complete removal of deposits and bacterial toxins. aPDT has been reported to result in additional improvement in clinical parameters of periodontal disease. It is supported by in vitro studies that report effective killing of periodontal microbes in planktonic and biofilm samples. ${ }^{16,17}$ Therefore, the aim of the present study was to evaluate the additional benefit of aPDT, if any, in addition to SRP alone.

At the baseline examination, there was no statistically significant difference between the two groups in any of the recorded parameters i.e. PI, mSBI, PD and CAL. All four parameters improved in both treatment groups after 1 month and 3 months post-treatment interval as observed in many previous studies. ${ }^{18-20}$

BOP has been emphasized as a predictor of periodontal disease, while its absence is even a more reliable indicator for the maintenance of periodontal health. ${ }^{21}$ Larger reduction observed in $\mathrm{mSBI}$ in $\mathrm{SRP}+\mathrm{aPDT}$ group, that was statistically significant at both 1 month and 3 months post treatment interval, was consistent with the studies of Alwaeli et al, ${ }^{8}$ Braun et al, ${ }^{9}$ Andersen et al, ${ }^{22}$ Chondros et $\mathrm{al}^{23}$ and Christodoulides et al. ${ }^{24}$ It can be seen in this study that aPDT has a positive effect on patient care, mainly due to the considerably fast resolution of overt inflammation in the gingival tissues, which is supported by the significant reduction in $\mathrm{mSBI}$. It is noteworthy that the reduc- 
tion in bleeding indices is the most consistent finding in almost all the clinical trials. ${ }^{25}$ A plausible explanation for this improvement in the SRP + aPDT group patients could be bacterial load reduction and inactivation of bacterial virulence factors and cytokines when the photosensitizer dye is irradiated with laser. ${ }^{26}$ Komerik et al successfully demonstrated killing of Porphyromonas gingivalis in the gingival crevices of rats without damaging the adjacent periodontal tissues. ${ }^{27}$ In another study, Pinheiro et al showed a significantly greater reduction in the percentage of viable bacteria in periodontal pockets treated with aPDT (96\%) compared to those treated with SRP alone $(81 \%) .{ }^{28}$ Another study by de Oliveira et al observed reduction in crevicular pro-inflammatory cytokines, tumour necrosis factor- $\alpha$ (TNF- $\alpha$ ) and receptor activator of nuclear factor kappa-b ligand (RANKL) levels in patients with periodontitis, subjected to aPDT. ${ }^{29}$

Significant reduction in PD during first month in both groups can be attributed to unintentional curettage and subsequent drainage of exudate that occurs commonly with sub-gingival instrumentation. Inter-group difference in reduction of PD at 1 month was statistically significant, in favour of the SRP + aPDT group. Higher reduction of $\mathrm{PD}$ in the SRP + aPDT group at 1 month may have resulted from additional shrinkage in the inflamed pocket wall after reduction in microbial load achieved by PDT.

The SRP + aPDT group again had an edge over SRP group at 3 months post treatment follow up, however, this time, the inter-group difference in $\mathrm{PD}$ reduction was statistically non-significant in accordance with studies by Chondros et al, ${ }^{23} \mathrm{Al}$-Zahrani et al, ${ }^{30}$ Polansky et al, ${ }^{31}$ and Rühling et al. ${ }^{32}$ In contrast, various other clinical trials have reported significantly higher reduction of $\mathrm{PD}$ in patients receiving aPDT in addition to SRP. ${ }^{9,33}$ However, their results cannot be compared with the present study as there were major differences in terms of study design, sample size, and frequency of aPDT application. Lulic et al repeated the PDT 5 times during the period of 6 months. ${ }^{33}$ Alwaeli et al, who used the same PDT system with the same settings as in our study, reported higher reduction in PD achieved with adjunctive use of PDT. However, they had evaluated 136 sites with CAL $\geq 4 \mathrm{~mm}$, versus 60 sites in our study. ${ }^{8}$

The assessment of clinical effectiveness of aPDT on the basis of PD can be misleading, as the manual probing pressure can vary at different times and in different patients leading to some errors in measurements. Additionally, the disease activity of periodontal pockets might be overlooked in the shadow of calculating such mean values. Since BOP has been found to be the most consistent indicator of present disease activity, ${ }^{21}$ changes in mean values of PD cannot be over emphasized, especially in case of limited sample size. ${ }^{34,35}$

CAL improved significantly in both groups, however, inter-group difference was not significant either at 1 month or 3 months. Similar results were reported by many previous studies. ${ }^{9,23,24,30}$ Our findings confirm the results of a recent study by Balata et $\mathrm{al}^{36}$ who reported that aPDT did not add any additional effect to SRP in terms of CAL gain.
They had evaluated the parameters in deep periodontal pockets ( $P D \geq 7 \mathrm{~mm}$ ), similar to our study. However, the sample size was smaller (22 subjects), and different photosensitizer along with different laser system were used. Since aPDT functions primarily at decreasing the microbial counts, these results are suggestive of no additional role of aPDT in improvement of CAL.

Our results also confirm those reported by Chondros et $\mathrm{al},{ }^{23} \mathrm{Christodoulides}$ et $\mathrm{al},{ }^{24}$ and $\mathrm{Ge}$ et $\mathrm{al},{ }^{37}$ i.e. significant difference in reduction of bleeding indices while CAL improved equally in both test and control groups. However, the study by Christodoulides et $\mathrm{al}^{24}$ was of parallel design instead of split mouth. Inclusion of smokers in the study by Chondros et $\mathrm{al}^{23}$ should also be considered while comparing the results.

Mombelli et $\mathrm{al}^{38}$ observed that reduction of selected gram-negative anaerobic organisms in the sub gingival plaque is a more important element for the success of periodontal therapy than is the removal of contaminated root cementum and mineralized deposits by root planing. PDT as an antimicrobial modality ensured reduction in microbial challenge that might not had been cleared by mechanical instrumentation in deep pockets. Antimicrobial PDT has other advantages as well, like broad spectrum of antimicrobial effect, no development of resistance, easy application and no repetition being required until BOP reappears. Biofilms reduce the effectiveness of aPDT, but not as much as reported in cases of antibiotics. ${ }^{17}$

The results of the present study could have been more meaningful if the probing force had been calibrated. A larger sample size and a longer follow up time would further clarify the outcomes of this study. Lack of microbiological data was another limitation, as improvement in clinical parameters could have been better explained with changes in microbial spectrum. Since a single application of aPDT was done in the present study, it is unclear if multiple aPDT application would further improve the outcome of nonsurgical periodontal therapy, as reported previously by Lulic et al. ${ }^{33}$

\section{Conclusion}

Within the limitations of this study, it may be concluded that aPDT plays an additional role in reduction of gingival inflammation when used along with nonsurgical mechanical debridement in deep periodontal pockets. It may be recommended to use aPDT along with SRP for better improvements in gingival bleeding scores. However, since it has primarily antimicrobial action, its role in pocket depth reduction and clinical attachment gain appears to be limited.

\section{Ethical Considerations}

This study was approved by ethical committee of Maulana Azad Institute of Dental Sciences, New Delhi.

\section{Conflict of Interests}

The authors have no conflict of interest to declare. 


\section{References}

1. Jori G, Fabris C, Soncin M, et.al. Photodynamic therapy in the treatment of microbial infections: basic principles and perspective applications. Lasers Surg Med. 2006;38:468481. doi:10.1002/lsm.20361.

2. Meisel P, Kocher T. Photodynamic therapy for periodontal diseases: state of the art. J Photochem Photobiol. 2005;79:159-170. doi:10.1016/j.jphotobiol.2004.11.023.

3. Al-Zahrani MS, Obadah NA. Photodynamic therapy as an adjunctive to scaling and root planing in treatment of chronic periodontitis in smokers. Saudi Med J. 2011;32:1183-1188.

4. Umeda M, Takeuchi Y, Noguchi K, Huang Y, Koshy G, Ishikawa I. Effects of nonsurgical periodontal therapy on the microbiota. Periodontol 2000. 2004;36:98-120. doi:10.1111/j.1600-0757.2004.03675.x.

5. Walker CB. The acquisition of antibiotic resistance in the periodontal microflora. Periodontol 2000. 1996;10:79-88. doi:10.1111/j.1600-0757.1996.tb00069.x

6. Socransky SS, Haffajee AD. Dental biofilms: Difficult therapeutic targets. Periodontol 2000. 2002;28:12-55. doi:10.1034/j.1600-0757.2002.280102.x.

7. Wainwright M. Photodynamic antimicrobial chemotherapy (PACT). J Antimicrob Chemother. 1998;42:13-28. doi:10.1093/jac/42.1.13.

8. Alwaeli HA, Al-Khateeb SN, Al-Sadi A. Long-term clinical effect of adjunctive antimicrobial photodynamic therapy in periodontal treatment: a randomized clinical trial. Lasers Med Sci. 2015;30: 801-807. doi:10.1007/s10103-013-1426-y.

9. Braun A, Dehn C, Krause F, Jepsen S. Short-term clinical effects of adjunctive antimicrobial photodynamic therapy in periodontal treatment: a randomized clinical trial. $J$ Clin Periodontol. 2008;35:877-884. doi:10.1111/j.1600051x.2008.01303.x.

10. Sbordone L, Bortolaia C. Oral microbial biofilms and plaque-related diseases: microbial communities and their role in the shift from oral health to disease. Clin Oral Investig. 2003;7:181-188. doi:10.1007/s00784-0030236-1.

11. Haffajee AD, Teles RP, Socransky SS. The effect of periodontal therapy on the composition of the subgingival microbiota. Periodontol 2000. 2006;42:219-258. doi:10.1111/j.1600-0757.2006.00191.x.

12. Demidova TN, Hamblin MR. Effect of cell-photosensitizer binding and cell density on microbial photoinactivation. Antimicrob Agents Chemother. 2005;49:2329-2335. doi:10.1128/aac.49.6.2329-2335.2005.

13. Ahad A, Tandon S, Lamba AK, Faraz F. Adjunctive use of antimicrobial photodynamic therapy in periodontal treatment of a patient susceptible to osteoradionecrosis. J Cranio Max Dis. 2015;4:157-162. doi:10.4103/22789588.163267.

14. Dahle'n G, Lindhe J, Sato K, Hanamura H, Okamoto $\mathrm{H}$. The effect of supragingival plaque control on the subgingival microbiota in subjects with periodontal disease. J Clin Periodontol. 1992;19:802-809. doi:10.1111/ j.1600-051x.1992.tb02174.x.

15. McNabb H, Mombelli A, Lang NP. Supragingival cleaning 3 times a week. J Clin Periodontol. 1992;19:348-356. doi:10.1111/j.1600-051x.1992.tb00658.x.

16. Song HH, Lee JK, Um HS, Chang BS, Lee SY, Lee MK. Phototoxic effect of blue light on the planktonic and biofilm state of anaerobic periodontal pathogens. J Periodontal
Implant Sci. 2013;43:72-78.

17. Fontana CR, Abernethy AD, Som S, et al. The antibacterial effect of photodynamic therapy in dental plaque-derived biofilms. J Periodontal Res. 2009;44:751-759. doi:10.1111/ j.1600-0765.2008.01187.x.

18. Badersten A, Nilveus R, Egelberg J. Scores of plaque, bleeding, suppuration and probing depth to predict probing attachment loss. 5 years of observation following nonsurgical periodontal therapy. J Clin Periodontol. 1990;17:102-107. doi:10.1111/j.1600-051x.1990.tb01070.x.

19. Lindhe J, Nyman S. The effect of plaque control and surgical pocket elimination on the establishment and maintenance of periodontal health. A longitudinal study of periodontal therapy in cases of advanced disease. J Clin Periodontol. 1975;2:67-79. doi:10.1111/j.1600-051x.1975.tb01727.x.

20. Lindhe J, Westfelt E, Nyman S, Socransky SS, Haffajee AD. Long-term effect of surgical/non-surgical treatment of periodontal disease. J Clin Periodontol. 1984;11:448-458. doi:10.1111/j.1600-051x.1984.tb01344.x.

21. Lang NP, Adler R, Joss A, Nyman S. Absence of bleeding on probing an indicator of periodontal stability. J Clin Periodontol. 1990;17:714-721. doi:10.1111/j.1600051x.1990.tb01059.x.

22. Andersen R, Loebel N, Hammond D, Wilson M. Treatment of periodontal disease by photodisinfection compared to scaling and root planing. J Clin Dent. 2007;18:34-38.

23. Chondros P, Nikolidaki D, Christodoulides N, Ro“ssler R, Gutknecht N, Sculean A. Photodynamic therapy as adjunct to non-surgical periodontal treatment in patients on periodontal maintenance: A randomized controlled clinical trial. Lasers Med Sci. 2009;24:681-688. doi:10.1007/ s10103-008-0565-z.

24. Christodoulides N, Nikolidakis D, Chondros P, et al Photodynamic therapy as an adjunct to non-surgical periodontal treatment: a randomized, controlled clinical trial. J Periodontol. 2008;79:1638-1644. doi:10.1902/ jop.2008.070652.

25. Sgolastra F, Petrucci A, Gatto R, Marzo G, Monaco A. Photodynamic therapy in the treatment of chronic periodontitis: a systematic review and meta-analysis. Lasers Med Sci. 2013; 28:669-682. doi:10.1007/s10103-0111002-2.

26. Braham P, Herron C, Street C, Darveau R. Antimicrobial photodynamic therapy may promote periodontal healing through multiple mechanisms. J Periodontol. 2009;80:17901798. doi:10.1902/jop.2009.090214.

27. Komerik N, Nakanishi H, MacRobert AJ, Henderson B, Speight P, Wilson M. In vivo killing of Porphyromonas gingivalis by toluidine blue-mediated photosensitization in an animal model. Antimicrob Agents Chemother. 2003;47:932-940. doi:10.1128/aac.47.3.932-940.2003.

28. Pinheiro SL, Donega JM, Seabra LM, et al. Capacity of photodynamic therapy for microbial reduction in periodontal pockets. Lasers Med Sci. 2010;25:87-91. doi:10.1007/s10103-009-0671-6.

29. de Oliveira RR, Schwartz-Filho H, Novaes A Jr. Antimicrobial photodynamic therapy in the non-surgical treatment of aggressive periodontitis: cytokine profile in gingival crevicular fluid, preliminary results. J Periodontol. 2009;80:98-105. doi:10.1902/jop.2009.070465.

30. Al-Zahrani MS, Bamshmous SO, Alhassani MA, AlSherbini MM. Short-term effects of photodynamic therapy on periodontal status and glycemic control of patients with 
diabetes. J Periodontol. 2009;80:1568-1573. doi:10.1902/ jop.2009.090206

31. Polansky R, Haas M, Heschl A, Wimmer G. Clinical effectiveness of photodynamic therapy in the treatment of periodontitis. J Clin Periodontol. 2009;36:575-580. doi:10.1111/j.1600-051x.2009.01412.x.

32. Rühling A, Fanghänel J, Houshmand M, et al. Photodynamic therapy of persistent pockets in maintenance patients-a clinical study. Clin Oral Invest. 2010;14:637-644. doi:10.1007/s00784-009-0347-4.

33. Lulic M, Leigegener GI, Salvi GE, Ramseier CA, Mattheos N, Lang NP. One-year outcomes of repeated adjunctive photodynamic therapy during periodontal maintenance: a proof-of-principle randomized-controlled clinical trial. J Clin Periodontol. 2009;36:661-666. doi:10.1111/j.1600051x.2009.01432.x.

34. Axelsson, P, Lindhe J. The significance of maintenance care in the treatment of periodontal disease. J Clin Periodontol. 1981;8:281-294. doi:10.1111/j.1600-051x.1981.tb02039.x.
35. Heitz-Mayfield LJ, Trombelli L, Heitz F, Needleman I, Moles D. A systematic review of the effect of surgical debridement $v s$ non-surgical debridement for the treatment of chronic periodontitis. J Clinical Periodontol. 2002;29:92-102. doi:10.1034/j.1600-051x.29.s3.5.x.

36. Balata ML, de Andrade LP, Santos DB., Cavalcanti AN, Ribeiro ED, Bittencourt S. Photodynamic therapy associated with full-mouth ultrasonic debridement in the treatment of severe chronic periodontitis: randomizedcontrolled clinical trial. J Applied Oral Sci. 2013;21:208214. doi:10.1590/1678-7757201302366.

37. Ge L, Shu R, Li Y, et al. Adjunctive effect of photodynamic therapy to scaling and root planing in the treatment of chronic periodontitis. Photomed Laser Surg. 2011;29:33-37. doi:10.1089/pho.2009.2727.

38. Mombelli A, Marxer M, Gaberthüel T, Grunder U, Lang NP. The microbiota of osseointegrated implants in patients with a history of periodontal disease. J Clin Periodontol. 1995;22:124-130. doi:10.1111/j.1600-051x.1995.tb00123.x. 